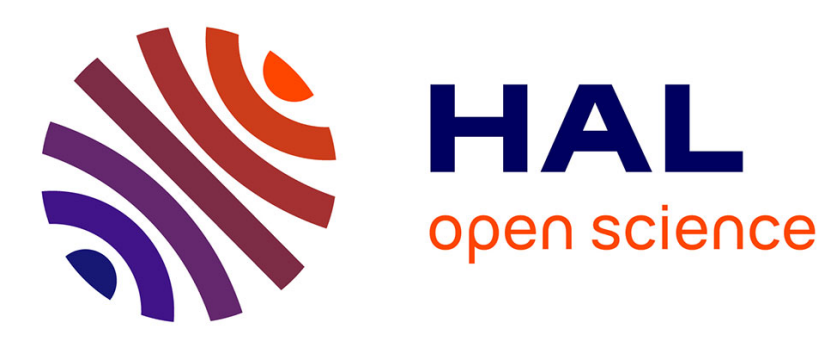

\title{
Histoire et mémoires des immigrations en régions aux XIXe et XXe siècle
}

\author{
Anne Sophie Bruno, Gérard Noiriel, Laure Pitti, Philippe Rygiel, Yann \\ Scioldo-Zürcher, Alexis Spire, Claire Zalc
}

\section{- To cite this version:}

Anne Sophie Bruno, Gérard Noiriel, Laure Pitti, Philippe Rygiel, Yann Scioldo-Zürcher, et al.. Histoire et mémoires des immigrations en régions aux XIXe et XXe siècle. Hommes \& migrations, 2008, Histoires des immigrations. Panorama régional, 1273, pp.6-17. 10.3406/homig.2008.4729 . halshs01353869

\section{HAL Id: halshs-01353869 \\ https://shs.hal.science/halshs-01353869}

Submitted on 15 Aug 2016

HAL is a multi-disciplinary open access archive for the deposit and dissemination of scientific research documents, whether they are published or not. The documents may come from teaching and research institutions in France or abroad, or from public or private research centers.
L'archive ouverte pluridisciplinaire HAL, est destinée au dépôt et à la diffusion de documents scientifiques de niveau recherche, publiés ou non, émanant des établissements d'enseignement et de recherche français ou étrangers, des laboratoires publics ou privés. 


\title{
Histoire et mémoires des immigrations en régions auX $X I X$ et $X X$ siècles
}

\author{
Par Anne-Sophie Bruno, Gérard Noiriel, Laure Pitti, \\ Philippe Rygiel, Yann Scioldo-Zürcher, Alexis Spire, Claire Zalc, \\ Coordination nationale de l'enquête ${ }^{(1)}$
}

Il y a quelques mois se terminait la première phase du programme de recherche "Histoire et mémoires des immigrations en régions aux XIX et XX ${ }^{e}$ siècles", initié en 2005 par l'Agence nationale pour la cohésion sociale et l'égalité des chances (l'Acsé). La coordination scientifique chargée de mettre en ceuvre sa réalisation dans dix régions de France métropolitaine avait un double objectif : renouveler les connaissances sur le fait migratoire et donner aux acteurs locaux comme aux habitants les moyens de prendre en charge leur propre mémoire régionale.

Les résultats de la première tranche de cette étude répondent aux objectifs qui avaient été fixés : ils procurent à tous un ensemble d'outils archivistiques, statistiques et historiographiques et renouvellent le regard sur une histoire de limmigration jusqu'alors étudiée surtout dans un cadre national.

\section{Richesse et originalité du matériel d'enquête}

Les précieux guides des sources établis par l'association Génériques datant de $1999^{(2)}$, il a fallu les compléter par les innombrables cotes résultant des nouveaux classements effectués par les différents centres de conservation des archives nationales et départementales.

Le travail réalisé n'a cependant pas été qu'une simple mise à jour : il a aussi fait apparaitre des fonds nouveaux. On a ainsi pu constater que même les régions $a$ priori peu concernées par l'immigration recelaient d'importants fonds - comme les régions Bretagne et Picardie notamment. 
L'originalité des inventaires dressés réside aussi dans la diversité des séries mobilisées. On note, par exemple, de précieuses informations sur la pénalisation de l'immigration dans les inventaires des jugements correctionnels des tribunaux de Laon et de Soisson.

Le rapport sur l'Alsace mentionne pour sa part le Centre rhénan d'archives et de recherches économiques (Cerare), basé à Mulhouse, qui conserve, depuis le milieu des années quatre-vingt, des archives d'entreprises complémentaires de celles que conserve le Centre des archives du monde du travail de Roubaix.

Différents rapports évoquent également les archives municipales. Notons que les communes d'Alsace-Moselle, selon une caractéristique héritée de la période allemande, enregistrent jusque dans les années soixante l'état civil, la situation familiale, l'adresse, la profession et la religion de leurs habitants.

D'autres fonds, plus inattendus, ont parfois aussi été visités, comme ceux des chaines régionales de télévision, de musées nationaux et locaux, d'hôpitaux et d'universités. Certaines équipes ont enfin exploré les archives privées - syndicats, partis politiques, groupes religieux, associations et "personnalités".

Les études sur les régions Nord - Pas-de-Calais, Bretagne et Midi-Pyrénées ont aussi développé une approche filmographique offrant des pistes de recherches nouvelles sur les représentations de l'immigration.

Tout en offrant des matériaux nouveaux, les travaux engagés fournissent un tableau de l'historiographie régionale.

\section{Une abondante historiographie locale}

La plupart des rapports se sont appuyés sur la recension des mémoires d'étudiants conservés dans les universités, donnant aux chercheurs l'opportunité, rare, de mettre en relation des travaux difficiles à localiser et souvent lacunaires. L'étude de ces recherches universitaires a permis également de savoir à quel rythme se sont développés les travaux sur l'immigration selon les différentes unités de recherche.

Si les études sur l'immigration sont nombreuses dans toutes les régions, plusieurs facteurs conditionnent leur production : l'importance et l'ancienneté des migrations, le dynamisme des institutions locales - universitaires ou non -, les questionnements individuels ou collectifs, ainsi que l'importance des imaginaires associés à certains groupes de migrants. On constate que si les impulsions données par les grands travaux sur l'historiographie de l'immigration ne sont pas toujours adaptées aux cadres locaux et régionaux, elles ont néanmoins eu des répercussions. 
La construction de l'histoire de l'immigration tient en premier lieu à la présence étrangère. Une immigration faible, récente et dispersée, n'offre pas aux chercheurs la profondeur historique et les effets de masse encourageant à s'y intéresser - quoique l'intérêt porté à un groupe de migrants ne soit pas forcément proportionnel à son nombre. Aucune étude n'a ainsi été menée dans les Pays de la Loire sur les migrants algériens, portugais, marocains et britanniques, alors que l'on dénombre des mémoires universitaires portant sur les migrants libanais, japonais, croates et ukrainiens.

Inversement, la forte présence d'immigrés a impulsé des travaux de recherche bien avant la naissance d'une historiographie nationale. Si les travaux menés en région Midi-Pyrénées sur l'immigration espagnole sont nombreux, ils témoignent cependant d'un faible intérêt pour une immigration "hors exil" - pourtant ancienne et structurée depuis le Second Empire.

On note aussi un intérêt très marqué pour l'histoire migratoire italienne. C'est le cas en Normandie, alors que les Italiens y sont pourtant très peu nombreux. Cet intérêt résulte surtout de la force des travaux de Pierre Milza, l'un des pionniers de cette histoire spécifique.

L'étude de cette historiographie régionale montre que l'impulsion de travaux dépend non seulement des intérêts des chercheurs universitaires, mais aussi des types de formations dispensées. Une partie importante des mémoires sur l'immigration déposés à l'université Jules-Verne de Picardie provient ainsi de la direction de l'Éducation permanente et ont été soutenus dans le cadre de la maitrise "Travailleur social". Dans l'ensemble, ces travaux à mi-chemin entre formation professionnelle et formation universitaire ont été réalisés dans les années quatre-vingt, les mémoires strictement universitaires plutôt à partir des années quatre-vingt-dix.

\section{Une histoire dont la chronologie varie selon les régions}

Les données recueillies et les synthèses qui en ont été faites renouvellent également notre connaissance de l'histoire de l'immigration. Nous savons désormais que celle-ci ne se déroule pas selon la même chronologie dans les différentes régions et qu'elle est loin de revêtir partout les mêmes formes.

En Alsace par exemple, la présence étrangère est ancienne, antérieure à 1850, autour du pôle urbain de Mulhouse, dont l'industrialisation fut précoce. Dans la période de l'entre-deux-guerres et au cours des Trente Glorieuses, les flux migratoires furent en revanche plus modestes dans cette région. 
Inversement, la région Normandie est moins concernée par les vagues migratoires que l'ensemble du territoire national : elle se situe toujours nettement en dessous de la moyenne nationale du pourcentage d'étrangers présents et présente des décalages dans le temps et dans les rythmes d'installation.

En Midi-Pyrénées, durant les Trente Glorieuses, le nombre des arrivées d'étrangers est très inférieur à la moyenne nationale - sauf en Haute-Garonne, entraînée par Toulouse - et, jusqu'à la fin des années soixante, la population étrangère $y$ diminue constamment.

Quant au grand flux des années vingt, il est très amplifié dans le Nord - Pas-de-Calais : en 1931, le département du Nord est le troisième département français pour son nombre d'immigrés, le Pas-de-Calais le quatrième. À l'inverse, à partir de 1975, la région voit s'installer peu de nouveaux venus. Elle

\author{
Une réflexion autour \\ de la position frontalière \\ de certaines régions et \\ des migrations \\ spécifiques qui en \\ résultent semble une \\ autre piste intéressante \\ à développer.
} devient alors la neuvième région française pour la proportion d'étrangers. Un tel décalage entre moyennes nationales et régionales montre combien les rythmes et les structures de l'économie locale sont des déterminants fondamentaux en histoire migratoire : ici, le poids du décollage industriel de la région dans la première partie $\mathrm{du} \mathrm{XX}^{\mathrm{e}}$ siècle conditionne les arrivées, tandis que la crise violente des années soixante-dix entraîne le délitement de cette "tradition" migratoire.

Même chose en Lorraine où, à partir de 1895, les houillères, les mines de fer, la sidérurgie et le textile-habillement alimentent des flux importants jusque dans les années soixante.

L'approche statistique comparative n'est bien évidemment pas la seule source de savoirs possible et, à la lecture des différents rapports, une réflexion autour de la position frontalière de certaines régions et des migrations spécifiques qui en résultent semble une autre piste intéressante à développer.

\section{Les frontières nationales, des particularités régionales}

Cinq des régions étudiées ont une frontière avec un ou plusieurs pays. Une telle situation géographique peut induire de nombreux va-et-vient entre deux pays, voire une immigration pendulaire du fait de la présence d'un bassin d'emploi attractif. 
La frontière - que l'équipe de recherche qualifie "d'aimant frontalier" - est un élément essentiel de l'histoire migratoire du Nord - Pas-de-Calais - du moins jusqu'à la Première Guerre mondiale. Tout au long du XIx ${ }^{\mathrm{e}}$ siècle, on assiste à la transformation de migrations locales en immigration, par l'édification de la frontière nationale de 1815. Entre 1850 et 1914, le grand exode belge est lié, en partie, aux centres textiles.

La Lorraine est aussi caractérisée par d'importants mouvements transfrontaliers. À la fin du XIX ${ }^{e}$ siècle, ils sont en partie induits par l'annexion de l'Alsace-Moselle par l'Allemagne. Mais ces mouvements sont aussi liés au développement industriel, qui attire de nombreux travailleurs des pays voisins. Les Allemands représentent ainsi la première nationalité en Meurthe-et-Moselle jusqu'au boom économique du milieu des années 1890. Ces mouvements transfrontaliers, liés à l'activité économique, s'inversent à la fin du $\mathrm{Xx}^{\mathrm{e}}$ siècle, au moment où la sidérurgie entre en crise. On assiste alors, dès les années soixante, à un renversement des courants migratoires : les échanges de travail deviennent ainsi bénéficiaires pour l'Allemagne, puis pour le Luxembourg et la Belgique.

Les régions littorales, en revanche, ne semblent pas, à première vue, bénéficier de leur ouverture maritime. Il semble difficile d'imputer la présence d'Anglais en Normandie jusqu'en 1896 à la seule situation littorale de la région car, dès avant la Première Guerre mondiale, on y constate également la présence importante et atypique de populations espagnoles. Les régions possédant une façade maritime ne sont pas des régions frontalières. Cependant, la Normandie deviendra un lieu de passage, notamment vers les États-Unis. L'étude menée à partir des recensements communaux de la population étrangère dans la ville portuaire du Havre entre 1881 et 1926 montre une présence précoce - dès 1851 - et importante d'étrangers en partance pour les Amériques.

\section{Mains-d'œuvre industrielle et rurale}

Très diverses, les formes d'implantation des immigrants varient aussi en fonction des structures économiques régionales et de la répartition géographique des secteurs économiques employeurs de main-d'ceuvre immigrée.

En Lorraine, le développement de l'activité minière et sidérurgique recourt d'abord à une main-d'cuvre uniquement locale, ou transfrontalière, formée essentiellement d'Allemands. Durant la décennie 1890, le patronat local met en place des filières de recrutement de travailleurs italiens. Après la Première Guerre mondiale, on n'assiste à un retour de la main-d'ceuvre étrangère qu'au moment de 
la reprise économique des années vingt - mais elle n'est pas employée dans les activités de reconstruction, où prédominent les Français.

Lors de la crise des années trente, la diminution de la population active étrangère s'opère de manière tout aussi sélective : la crise industrielle touche surtout la métallurgie et la sidérurgie lourde, plus que le textile ou l'agriculture. Pourtant, elle ne s'accompagne pas d'une diminution systématique de la main-d'ceuvre étrangère dans les secteurs les plus touchés, le sort des travailleurs étrangers étant aussi tributaire de la politique menée par telle ou telle entreprise.

Les effets de la loi de 1932 sont ainsi limités en Lorraine, le patronat de la métallurgie étant parvenu à éviter la publication d'un décret restreignant la part de main-d'ceuvre étrangère dans son secteur d'activité jusqu'en 1936. La publication de ce décret changera cependant peu les choses, la loi des quarante heures venant alors bouleverser les règles $\mathrm{du}$ jeu. Le patronat lorrain adopte ainsi dans les années trente une position libérale visant à limiter l'encadrement de la main-d'cuvre étrangère par les autorités administratives, afin d'ajuster recrutements et licenciements en fonction de ses propres besoins.

À l'opposé de cette immigration industrielle, conforme aux représentations largement répandues de l'immigré ouvrier, d'autres régions ont connu une immigration principalement répartie autour des emplois du secteur primaire, puis du BTP et de la petite ou moyenne industrie.

En Picardie, le secteur agricole accueille $30 \%$ des actifs étrangers en 1901, et $63 \%$ en 1926. La région Midi-Pyrénées reçoit également une main-d'ceuvre étrangère principalement rurale. Au moment du tournant démographique des années vingt, elle est employée massivement dans l'agriculture, avec une diversité de statuts professionnels qui reflète la segmentation du marché du travail de ce secteur. On trouve des propriétaires dans le Gers ; les fermiers, les métayers et les ouvriers agricoles sont plus nombreux en Haute-Garonne. Une évolution s'amorce à compter de la fin des années cinquante avec l'essor de l'industrie et du bâtiment. L'immigration accompagne aussi les mutations de l'agriculture et l'évolution du marché du travail. Pendant les années soixante et soixante-dix, les saisonniers tendent à remplacer les ouvriers agricoles, tandis que le recours à une main-d'ceuvre clandestine devient structurel. D'autres régions - plus secondaires sur le plan migratoire - ayant accueilli une main-d'ceuvre agricole présentent un panorama des secteurs d'activité plus nuancé.

En Bretagne, le recours à l'immigration étrangère est destiné à pallier l'exode des Bretons dans les travaux des mines et des carrières.

En Normandie, on remarque une importante prolétarisation de la main-d'ceuvre en 1931 : 71 \% des actifs étrangers sont ouvriers. En 1946, des données plus 
précises permettent de faire état d'une surreprésentation de la main-d'cuvre étrangère dans l'agriculture, mais également dans les industries de transformation et les transports.

\section{Diverses autres typologies et approches}

La provenance des migrants diffère à chaque époque et selon les régions. En Nord - Pas-de-Calais, chaque période semble se caractériser par une importante concentration nationale. Il en est ainsi du "temps des Belges" - la moitié de la population de Roubaix en 1886 est née belge - : rappel en filigrane que la croissance de la population de la région est largement due au solde migratoire.

Dans l'entre-deux-guerres, on constate une très forte concentration de Polonais dans le bassin minier du Pas-de-Calais, dans le Valenciennois et le Douaisis. Après la Seconde Guerre mondiale et jusqu'en 1973, les migrations méditerranéennes remplacent celles d'Europe centrale. Enfin, une dernière vague d'arrivée est consécutive à la signature, en 1963, d'une convention entre la France et le Maroc. De même, la Picardie se caractérise par une immigration concentrée autour d'une nationalité : jusqu'au premier tiers du Xx $x^{e}$ siècle, les Belges sont surreprésentés ; à partir de 1931, les Polonais occupent le premier rang; les Marocains et les Portugais seront majoritaires lors de la troisième vague migratoire.

Les origines nationales des migrants sont au contraire beaucoup plus diversifiées en Normandie. Dans les années vingt, Italiens et Polonais s'y implantent, les Belges restant le groupe le plus nombreux. Après le second conflit mondial, on note une forte progression de la population algérienne, qui devient majoritaire dans le département de la Seine-Maritime. Dans les années soixante enfin, le nombre de Portugais augmente au point de dépasser celui des Espagnols en 1968. Dans les années quatre-vingt-dix, la diversification se poursuit avec les arrivées de populations turque et d'Afrique subsaharienne.

Certaines enquêtes adoptent une typologie centrée sur l'immigration individuelle de travailleurs versus l'immigration familiale. Il est cependant difficile d'évaluer le poids de l'immigration familiale en termes de flux, la procédure de regroupement familial n'ayant été juridiquement formalisée que très tardivement, au milieu des années soixante-dix. Pour la mesurer, il faut bien souvent s'en remettre aux données statistiques résultant des recensements.

Le rapport sur la région Picardie appréhende ainsi l'opposition entre immigration familiale et immigration de célibataires au moyen d'un très riche développement 
sur l'évolution des flux par genre. Une particularité mérite d'être ici soulignée : de 1911 à 1921, la population des hommes étrangers progresse beaucoup plus vite que celle des femmes étrangères - $10 \%$ pour les hommes contre $4 \%$ pour les femmes; mais, de 1921 à 1926, le nombre de femmes étrangères progresse plus vite que celui des hommes - 17,5\% par an contre $6,7 \%$ chez les hommes -, alors même qu'on se situe encore dans un contexte d'arrivées massives.

Ainsi, les migrations des femmes ne peuvent être lues exclusivement comme des migrations familiales : elles s'inscrivent dans une dynamique migratoire autonome, pas nécessairement liée à l'arrivée des hommes quelques années auparavant.

Le fort taux de célibat au lendemain de la guerre - en 1921, 43 \% des étrangers recensés en Picardie sont célibataires - pourrait laisser penser qu'un grand nombre de femmes sont d'abord arrivées comme travailleuses célibataires pour fonder ensuite des familles avec des migrants déjà arrivés : la proportion de célibataires tombe à $31 \%$ en 1936. On peut également évoquer une autre hypothèse pour expliquer la présence accrue de femmes étrangères : la croissance des effectifs observée en Picardie entre 1921 et 1926 proviendrait ainsi non pas d'une poussée de l'immigration, mais d'une augmentation du nombre de femmes

\section{L'un des principaux apports de ce programme de recherche est de démontrer que l'impact des processus d'immigration est très différencié selon les territoires.}

françaises ayant épousé des étrangers et pris la nationalité de leur époux : la loi de 1927 mettra fin à cette possibilité en maintenant dans leur nationalité d'origine les femmes françaises ayant épousé un mari étranger.

Enfin, on constate des formes de migrations spécifiques aux régions.

En Alsace, les migrations étudiantes sont anciennes et doivent être mises en relation avec une forte activité missionnaire, qui s'est précocement traduite par l'envoi à Strasbourg, à la faculté de théologie, de jeunes gens jugés prometteurs.

Dans les flux contemporains, anciens et actuels, on observe également l'importance des migrations de refuge.

L'ouest de la France, dont la Bretagne, est ainsi caractérisé jusqu'à la fin du $\mathrm{XX}^{\mathrm{e}}$ siècle par une faible présence étrangère mais par une forte proportion de réfugiés, selon une tradition bien ancrée depuis le début du XIX ${ }^{\mathrm{e}}$ siècle. Polonais de 1830, Italiens "de l'unité" et Espagnols carlistes y sont nombreux. Au $\mathrm{Xx}^{\mathrm{e}}$ siècle, la présence de centres d'accueil conduit, dans une logique de dispersion des réfugiés sur l'ensemble du territoire national, un nombre 
important d'étrangers vers la Bretagne. Dans les années trente, l'implantation de réfugiés espagnols dans les centres d'accueil bretons perpétue cette tradition, quoiqu'une faible part d'entre eux se soit définitivement implantée dans la région. Dans les Pays de la Loire, on note la présence de réfugiés originaires du Sud-Est asiatique, en lien notamment avec une politique d'établissement pilotée nationalement qui détermine le point de chute en fonction non pas d'impératifs économiques mais de l'existence de relais locaux - relais associatifs de prise en charge, notamment.

\section{Les échelles d'analyse : régionale, départementale, cantonale ou bassin d'emploi}

Les rapports présentés dans le présent numéro représentent aussi un cadre idéal pour interroger l'échelle d'analyse régionale. L'un des principaux apports de ce programme de recherche est de démontrer que l'impact des processus d'immigration est très différencié selon les territoires. Ce constat vaut sur plusieurs échelles, qu'elles soient régionales ou plus fines - départementale, cantonale, ou encore autour du bassin d'emploi.

Le bassin de Couëron, en Loire-Atlantique, s'inscrit ainsi directement dans la dynamique des années trente, avec une arrivée massive de Polonais par le biais de la Société générale d'immigration, alors que le reste du département n'est quasiment pas touché par l'immigration.

Le rapport sur la Normandie introduit une discussion sur la pertinence de prendre la région comme unité de recherche et d'histoire. De fait, cette région recouvre cinq départements et deux régions administratives (les Haute et BasseNormandie) - découpage qui a déterminé la conception de la plupart des travaux, souvent conçus indépendamment les uns des autres, voire de façon concurrente. Le rapport sur la Picardie discute également cette approche régionale : dès la fin du XIX siècle, l'Oise - notamment sa partie sud, tournée vers Paris - devient un foyer majeur d'immigration, l'Aisne et la Somme restant en marge de ces grandes dynamiques.

Le rapport Midi-Pyrénées témoigne quant à lui de l'importance d'une analyse à l'échelle départementale, pour mettre au jour les disparités d'une région fortement clivée entre la Haute-Garonne, marquée par l'attraction de Toulouse, et ses départements voisins, fortement et longtemps ruraux, comme le Lot ou le Tarn. 
Enfin, l'approche monographique de nombreux rapports apporte également des éléments d'explication des disparités locales. Une étude sur l'immigration subsaharienne en Normandie montre ainsi que deux modèles d'immigration s'opposent : la Basse-Normandie a reçu une population immigrée plutôt qualifiée et employée dans tous les secteurs ; la Haute-Normandie a attiré une population moins qualifiée, plus massive, travaillant notamment dans l'industrie automobile. La comparaison entre les villes normandes du Havre et de Bolbec entre 1926 et 1975 montre comment la main-d'ceuvre étrangère a pu servir non à la croissance d'un secteur mais à accompagner son déclin, en permettant dans les années de crise une reconversion des actifs français du textile dans d'autres secteurs.

Des points de comparaison s'imposent avec d'autres monographies locales, notamment celle du rapport sur le Nord - Pas-de-Calais, qui compare la situation des mineurs polonais et marocains à Arenberg entre 1921 et 1989, faisant apparaître un recrutement des Marocains en contrat à durée déterminée - ou la mise en place d'une main-d'ceuvre temporaire pour faciliter la fermeture des puits.

Indépendamment de la question des sources - avec le recensement pour Bolbec, ou, pour Arenberg, les registres des entrées et sorties de l'entreprise, conservés à l'Agence nationale pour la garantie des droits des mineurs -, la concordance entre ces deux études de cas concourt à une meilleure connaissance du rôle des étrangers sur le marché du travail en France.

\section{Les projets mémoriels : des méthodes d'investigation diversifiées}

Le dernier volet de l'enquête, pour lequel les équipes avaient une grande latitude de recherche, présente un ensemble hétérogène de projets mémoriels et de méthodes d'investigation.

Recueil de mémoires : trente entretiens ont été notamment conduits au Havre. Le rapport développe la méthodologie de l'entretien, les questions de l'ethnicité et de l'identité et propose un matériel intéressant à exploiter.

Autre approche : l'étude de la construction des mémoires en fonction de tel ou tel groupe. L'étude de la commémoration de la rafle du 11 septembre 1942 des juifs de Lens permet, par exemple, d'interroger le rapport entre mémoires locales et mémoires immigrées dans une configuration locale spécifique. 
"Ainsi, même si la mémoire de l'événement se transmet du côté des familles qui en ont été victimes, dans la sphère privée, cette mémoire ne bénéficie pendant longtemps d'aucune reconnaissance publique. Le rétrécissement de la communauté juive de Lens avec, dès les années soixante, la disparition et surtout le départ de la plupart de ses membres, explique pour partie ce phénomène. Mais celui-ci semble avant tout dû à la domination d'une appartenance et d'une image ouvrière et minière dans le bassin. Les Polonais catholiques, qui font aujourd'hui figure d'immigrés modèles', ont partagé cette appartenance et ont fini par devenir un élément légitime de cette image" ${ }^{(3)}$

Les immigrés maghrébins arrivés plus récemment dans le bassin du Nord - Pasde-Calais ont aussi été exclus de cette mémoire.

Certaines approches associent recueil d'histoires de vie et "actions de réappropriation mémorielle". C'est notamment le cas des ateliers d'histoire locale avec les populations des quartiers mis en place par l'université populaire et citoyenne de Roubaix, qui anime aussi des "cafés citoyens" autour de diverses thématiques liées à l'immigration.

Le rapport Midi-Pyrénées observe l'histoire du Fasild et de la mémoire en recensant les "actions mémoires" engagées par cette institution.

En Bretagne, le projet pédagogique "Émigrations-Immigrations : entre mémoire et histoire" du collège Les Chalais à Rennes met en ceuvre un échange entre générations, avec pour particularité de faire dialoguer mémoires des migrants étrangers et mémoires des migrants ruraux, dans une région où la mémoire collective est davantage empreinte de l'histoire de l'émigration bretonne que de celle de l'immigration. Il s'agit ainsi de faire connaître la réalité de l'immigration à des enfants dont l'histoire personnelle n'a pas nécessairement été marquée par une expérience de migration transfrontalière.

En Lorraine enfin, une étude sur la mémoire a été mise en place à partir d'une problématique originale, appuyée sur Paul Ricceur et Reinhardt Koselleck. Fondée sur l'exploitation d'un grand nombre d'entretiens, elle souligne en conclusion la juxtaposition des mémoires d'immigration dans la région pendant plusieurs décennies. Les auteurs insistent aussi à juste titre sur l'importance des repères spatiaux, notamment la cité et l'usine, comme cadres sociaux de la mémoire.

Ils ouvrent deux autres pistes qui mériteraient d'être approfondies : d'une part, la mémoire se conjugue toujours avec l'oubli - d'où la nécessité de s'interroger sur ceux qui n'ont pas laissé de traces ; d'autre part, l'expérience de l'immigration n'est qu'une dimension de l'expérience vécue - qui doit être combinée avec d'autres aspects de l'identité sociale, notamment le genre et le milieu socioprofessionnel. 


\section{Un domaine de recherche en plein essor}

Ce premier rendu d'enquête propose un matériel considérable, qu'il est d'autant plus souhaitable de rendre public qu'il s'enrichira bientôt des résultats du deuxième volet du programme, aujourd'hui arrivé à son terme.

Grâce à la mobilisation des équipes qui ont dû travailler dans un temps court, les résultats obtenus et les pistes ouvertes témoignent de la vitalité d'un domaine de recherche qui rend à l'immigration toute sa place dans l'histoire nationale, la diversité des environnements et des territoires au sein desquels elle se déploie étant désormais prise en compte.

\section{Notes}

1. Anne-Sophie Bruno, maître de conférences en Histoire, Université de Paris XIII, Gérard Noiriel, directeur d'études, EhESS, Laure Pitti, maitre de conférences, l'université de Paris VIII, Philippe Rygiel, maitre de conférences, université Paris I, Yann Scioldo-Zurcher, chargé de recherche au CNRS - Migrinter, Alexis Spire, chargé de recherche au CNRS université de Lille-II, Claire Zalc, chargée de recherche au CNRS IHMC.

2. Génériques, Les Étrangers en France, guide des sources d'archives publiques et privées XIX-XX' siècles, 3 tomes, GénériquesDirection des Archives de France, 1999, 2048 p.

3. Voir : Histoire et mémoire des immigrations dans le Nord - Pas-de-Calais, XIX'-XX' siècles, Judith Rainhorn (dir.), mai 2007, l'Acsé. Ou : L'enquête collective - "Faire crédit aux classes populaires. Habiller les mineurs à la croisée des immigrations au XXe siècle" - menée par plusieurs membres de l'équipe scientifique qui a participé à l'étude sur le Nord - Pas-de-Calais est très largement consacrée à l'étude de l'immigration juive polonaise à Lens et à ses rapports avec les mineurs polonais de confession catholique dans le bassin minier (ouvrage à paraitre en 2008). Martina Avanza, Gilles Laferté, "Dépasser la 'construction des identités' ? Identification, image sociale, appartenance", Genèses, n 61, 2005, p. 154-167 ; Nicolas Mariot et Claire Zalc, "Identifier, s'identifier : recensement, auto-déclarations et persécution des Juifs lensois (1940-1945)", Revue d'histoire moderne et contemporaine, vol. 54, n 3, juillet-septembre 2007, p. 91-117; Ou Martina Avanza, Gilles Laferté, Nicolas Mariot, Claire Zalc, "Habiller les mineurs : une entreprise entre deux 'communautés' à Lens dans l'après-guerre", in Les Marges de la mine, Presses de l'université de Valenciennes (à paraître en 2008). 